\title{
Determination of indirect reference intervals of vitamin B12 for outpatients admitted to primary healthcare centers
}

https://doi.org/10.1515/labmed-2018-0160

Received June 9, 2018; accepted September 21, 2019; previously published online October 25, 2019

\begin{abstract}
Background: Vitamin B12 deficiency is still an important nutritional problem worldwide. There is no consensus on the reference intervals (RIs) for vitamin B12. In order to establish vitamin B12 RIs, we used the database obtained from a primary healthcare organization and estimated region-specific intervals.
\end{abstract}

Methods: Vitamin B12 results were collected from our laboratory information system (LIS) between January 2014 and July 2014. We excluded the results of the individuals who had megaloblastic and microcytic anemia. After outlier detection and exclusion, we estimated the RIs of the "1-12, 13-18, 19-64 and 65+" age groups according to the International Federation of Clinical Chemistry (IFCC) method. Statistical differences were examined between groups.

Results: The reference limits and 90\% confidence intervals (CIs) ( $n=14,748,3790$ male, age: 1-96; 10,958 female, age: 1-115) were estimated with 90\% CIs as 106 (104-107)-393 (391-395) pmol/L. We also estimated the reference limits of the " $1-12,13-18,19-64$ and $65+$ " age groups to be 127-459, 108-398, 106-384 and 104-426 pmol/L, respectively. There was no difference between genders in all age groups.

Conclusions: Both the lower and upper limits of the estimated RIs were found to be lower than the reference limits recommended by the manufacturer. Our findings revealed that the RIs of vitamin B12 should be given for the " $1-12$, 13-18, 19-64 and 65+" age groups separately. The big data obtained from laboratories should be used for the

\footnotetext{
*Correspondence: Esin Avci, Department of Medical Biochemistry, Faculty of Medicine, Pamukkale University, Kinikli, Pamukkale, Denizli 20020, Turkey, Phone: +00905337427123 ,

E-Mail: hekimesin@gmail.com

Diler Aslan: Department of Medical Biochemistry, Faculty of Medicine, Pamukkale University, Denizli, Turkey
}

determination of its population health status. As our population is composed of outpatients, the estimated vitamin B12 RIs may establish the basic information for the examination of our population for vitamin B12 deficiency.

Keywords: deficiency; primary health care; reference intervals; vitamin B12 deficiency.

\section{Introduction}

Laboratories are big data sources. Big data provide valuable information in terms of making a true diagnosis, appropriate treatment and reliable monitoring of a patient. It challenges the traditional approach to organizing and interpreting data [1, 2]. Unreliable and incorrect reference intervals (RIs) cause misdiagnosis and unnecessary treatments. Both upper and lower limits are important to decide the treatment models and timing. Therefore, RIs are of critical importance for the laboratory scientist [3]. Because estimating RIs is compulsory, laboratories mostly use manufacturer suggestions. Nevertheless, the laboratory specialist should evaluate patients' results with feedbacks from clinicians and estimate the local RIs at intervals by using an indirect approach. The main limitation of the indirect method is to include possible effects of diseased subpopulations on the derived interval [4]. Vitamin B12 is a water-soluble vitamin, which plays an important role in the functioning of the brain and nervous system, and in the formation of red blood cells. Vitamin B12 is involved in the metabolism of every cell in the body. It plays a role in the formation of DNA. It is needed not only for the synthesis and regulation of fatty acids but also for energy production [5]. The major causes of vitamin B12 deficiency are improper diet, malabsorption and alcoholism, with megaloblastic anemia being one of the serious results. Early diagnosis is essential as a deficiency of this vitamin can lead to irreversible neurological damage [6]. It is known that laboratory medicine data influence $70 \%$ of clinical decisions [3]. In this context, laboratory data 
can be used as an instrument for the determination of health and/or the disease management status. The laboratory data that is generated by the public health laboratories (PHLs) in Turkey can be considered as data centers for the determination of the health status of the population who live in provinces that the PHLs serve. Eightyone PHLs in 81 provinces in Turkey provide primary care laboratory services. Our laboratory is located in the center of the city whose population is 180,000 and serves 21 family health centers (FHCs) localized in different regions of the city. The purpose of our study was to estimate the RIs by using patients' test results in order to provide the vitamin B12 status of the population admitted to 21 FHCs to primary care physicians working at these centers, and a basis for further examination of our population.

\section{Materials and methods}

\section{Study group}

Vitamin B12 results were collected from the Uşak PHL information system between January 2014 and July 2014 in Uşak city in the western part of Turkey. The specimens collected from individuals admitted to the FHCs were analyzed in the PHL. We excluded patients with megaloblastic and microcytic anemia, and only the first measurement values of individuals who had repeated measurements determined according to their identity numbers were included. The Non-Interventional Medical Ethical Committee of Pamukkale University, Faculty of Medicine approved the study protocol.

\section{Analytical system}

The measurements were performed following the manufacturer's package inserts for Abbott reagents on an Architect i2000 sr analyzer (Abbott Diagnostics, Abbott Park, IL, USA). Three levels of internal quality control (Technopath Multichem IA Plus, IL, USA) were conducted twice a week with target values of 238, 336 and $448 \mathrm{pmol} / \mathrm{L}$. For a 6-month period, the intraassay and interassay coefficients of variability (CVs) were $<6 \%$ with average biases of $5.88 \%, 3.86 \%$ and $1.1 \%$, respectively.

We performed internal quality twice a week; only on Mondays and Thursdays, we analyzed vitamin B12 samples, but we accepted samples every day. After centrifugation, we stored the samples at $-20{ }^{\circ} \mathrm{C}$ until analysis.
We analyzed external quality samples using the external quality assessment scheme (EQAS) Biorad once a month. For vitamin B12, the 6-month EQAS CV results were $<5 \%$.

The limit of blank (LoB), limit of detection (LoD) and limit of quantitation (LoQ) were $92 \mathrm{pmol} / \mathrm{L}$ and LoB was $61 \mathrm{pmol} / \mathrm{L}$, respectively. The expected RI by the manufacturer was $138-652 \mathrm{pmol} / \mathrm{L}$.

\section{Estimation of reference intervals and statistics}

The histograms of all subgroups were inspected visually. Outliers were detected according to Tukey's method [7]. The distribution of each age cohort was analyzed for normality using the Kolmogorov-Smirnov (K-S) test.

According to the findings from the K-S test, the RIs of the groups were estimated by parametric or nonparametric methods according to the IFCC recommendations [8].

Partitioning was assessed according to the subgroup distributions. The decisions for separate RIs for the subgroups were made according the Lahti method for the Gaussian distribution or the nonparametric method (Kruskal-Wallis) for non-Gaussian distributions [9]. We also estimated the RIs of the whole group by using the Bhattacharya and Hoffman methods for approaching our data with different statistical methods [4].

The analysis of data was performed using SPSS Statistical Packages (version 21.0 for Windows, IBM Corporation 1989, 2012, Armonk, NY, USA).

\section{Results}

The mean, standard deviation (SD) and minimum-maximum values before and after outlier exclusion are shown in Table 1.

The distribution was found to be nonparametric according to K-analysis $(\mathrm{p}=0.0001)$. The subgroups were also found to be nonparametric with the $\mathrm{p}$-values for the subgroups starting from the 1-12 age group to $13-18$, 19-64 and 65+. There was no difference between genders in all age groups according to Kruskal-Wallis $(\mathrm{p}=0.659)$. The differences between the "1-12 and 19-64", "13-18 and 65+" and "1-12 and 13-18" age groups were found to be significant ( $p=0.0001$ for all subgroups) according to the three estimation methods. The RIs of these age groups were estimated by the nonparametric, Bhattacharya and Hoffman methods as shown in Table 2. We could not find any significant difference between the RIs of the "13-18 
Table 1: Laboratory data before $(n=15,455)$ and after $(n=14,748)$ outlier exclusion.

\begin{tabular}{|c|c|c|c|c|c|c|}
\hline & \multicolumn{3}{|c|}{ Before outlier exclusion } & \multicolumn{3}{|c|}{ After outlier exclusion } \\
\hline & Mean & SD & Min-Max & Mean & SD & Min-Max \\
\hline Hemoglobin, g/dL & 13.3 & 1.69 & $4.5-21.3$ & 13.3 & 1.69 & $4.5-21.3$ \\
\hline Hematocrit, \% & 39.1 & 4.52 & $17.6-60.5$ & 39.1 & 4.52 & $17.6-60.5$ \\
\hline $\mathrm{MCV}, \mathrm{fL}$ & 83.4 & 6.86 & $51-118$ & 83.4 & 6.83 & $53-118$ \\
\hline $\mathrm{MCH}, \mathrm{pg}$ & 28.3 & 2.83 & $14.8-49.4$ & 28.3 & 2.82 & $14.8-49.4$ \\
\hline $\mathrm{MCHC}, \mathrm{g} / \mathrm{dL}$ & 33.8 & 1.36 & $23-53$ & 33.9 & 1.36 & $23-53$ \\
\hline Red blood cell, $\mathrm{M} / \mu \mathrm{L}$ & 4.7 & 0.49 & $2.24-7.26$ & 4.7 & 0.49 & $2.24-7.26$ \\
\hline RDW-SD, fL & 19.0 & 11.1 & $11.1-62.8$ & 19 & 11.1 & $11.1-62.8$ \\
\hline Vitamin B12, pmol/L & 259 & 195 & $93-4221$ & 223 & 81.7 & $93-474$ \\
\hline
\end{tabular}

$\mathrm{MCH}$, mean corpuscular hemoglobin; MCHC, mean corpuscular hemoglobin concentration; MCV, mean corpuscular volume; RDW, red cell distribution width; SD, standard deviation.

Table 2: Serum vitamin B12, in the age groups and in the entire sample.

\begin{tabular}{|c|c|c|c|c|}
\hline \multirow[t]{4}{*}{ Age, years } & \multirow[t]{4}{*}{ Gender } & \multicolumn{3}{|c|}{ Reference interval vitamin B12, pmol/L } \\
\hline & & Nonparametric & Bhattacharya & Hoffman \\
\hline & & Lower $(90 \% \mathrm{Cl}) /$ upper $(90 \% \mathrm{Cl})$ & $-2 \mathrm{SD}(90 \% \mathrm{Cl}) /+2 \mathrm{SD}(90 \% \mathrm{Cl})$ & $-2 \mathrm{SD}(90 \% \mathrm{Cl}) /+2 \mathrm{SD}(90 \% \mathrm{Cl})$ \\
\hline & & Number of patients & Number of patients & Number of patients \\
\hline \multirow[t]{2}{*}{$1-12$} & $F \& M$ & $127(114-140) / 459(446-473)$ & $124(113-135) / 406(396-417)$ & $130(117-141) / 438(425-451)$ \\
\hline & & 345 & 349 & 336 \\
\hline \multirow[t]{2}{*}{$13-18$} & $F \& M$ & $108(100-117) / 398(395-406)$ & $127(121-133) / 338(332-344)$ & $108(101-116) / 359(351-367)$ \\
\hline & & 599 & 583 & 571 \\
\hline \multirow[t]{2}{*}{$19-64$} & $F \& M$ & $106(104-107) / 384(383-386)$ & $123(122-125) / 392(390-394)$ & $106(104-107) / 355(353-357)$ \\
\hline & & 11,427 & 11,270 & 10,737 \\
\hline \multirow[t]{2}{*}{$65+$} & $F \& M$ & $104(99-109) / 426(424-431)$ & $92(88-96) / 363(359-367)$ & $104(99-109) / 372(104-372)$ \\
\hline & & 2123 & 2055 & 2003 \\
\hline \multirow[t]{2}{*}{ All } & & $106(104-107) / 393(391-395)$ & $109(108-111) / 376(374-377)$ & $111(110-113) / 391(389-393)$ \\
\hline & & 14,478 & 14,290 & 13,211 \\
\hline
\end{tabular}

$\mathrm{Cl}$, confidence interval; $\mathrm{F}$, female; $\mathrm{M}$, male; SD, standard deviation.

and 19-64", "13-18 and 65+" and "19-64 and 65 +" groups with p-values of $0.069,0.509$ and 1.000 , respectively.

The reference limits and 90\% confidence intervals (CIs) $(\mathrm{n}=14,748,3790$ male, age: 1-96; 10,958 female, age: 1-115) were estimated to be 106 (104-107)-393 (391-395) pmol/L.

The graphical representation of the vitamin B12 test results of all individuals after the outlier exclusion are shown in Figure 1. The percentages of the vitamin B12-deficient population according to the manufacturer's cutoff level were determined to be $7.97 \%, 21.7 \%, 23.2 \%$, $24.6 \%$ and 23.2 for the $1-12,13-18,19-64,65+$ age groups, and the whole group, respectively.

\section{Discussion}

As a PHL, we decided to determine the RIs of vitamin B12 by the indirect method from the large amount of test results.
The indirect method for the estimation of RIs is suggested especially if a large amount of test results can be obtained [10]. In this context, we decided to estimate the indirect RIs. We were using the RIs suggested by the manufacturer as 138-652 pmol/L. After data collection from the laboratory information system (LIS), at first we stratified the data into the age and gender groups. We had 14,748 results for the estimation of vitamin B12 RIs after exclusion of the results according to our exclusion criteria. We had enough data, and the age groups were divided as 1-12, 13-18, 19-64 and $65+$ years. There were no differences between genders in all age groups. On the contrary, Akin [11], Papandreou [12], Wahlin [13], Demirin [14] and their colleagues reported vitamin RIs with age groups and genders in their RI studies. There was an article published without discrimination of age and gender in Turkey [15]. Özarda, Bakan and Tanyalçın reported vitamin B12 RIs with two genders but without age discrimination similar to our study [16-18]. We have summarized the vitamin B12 RI studies in Table 3. 

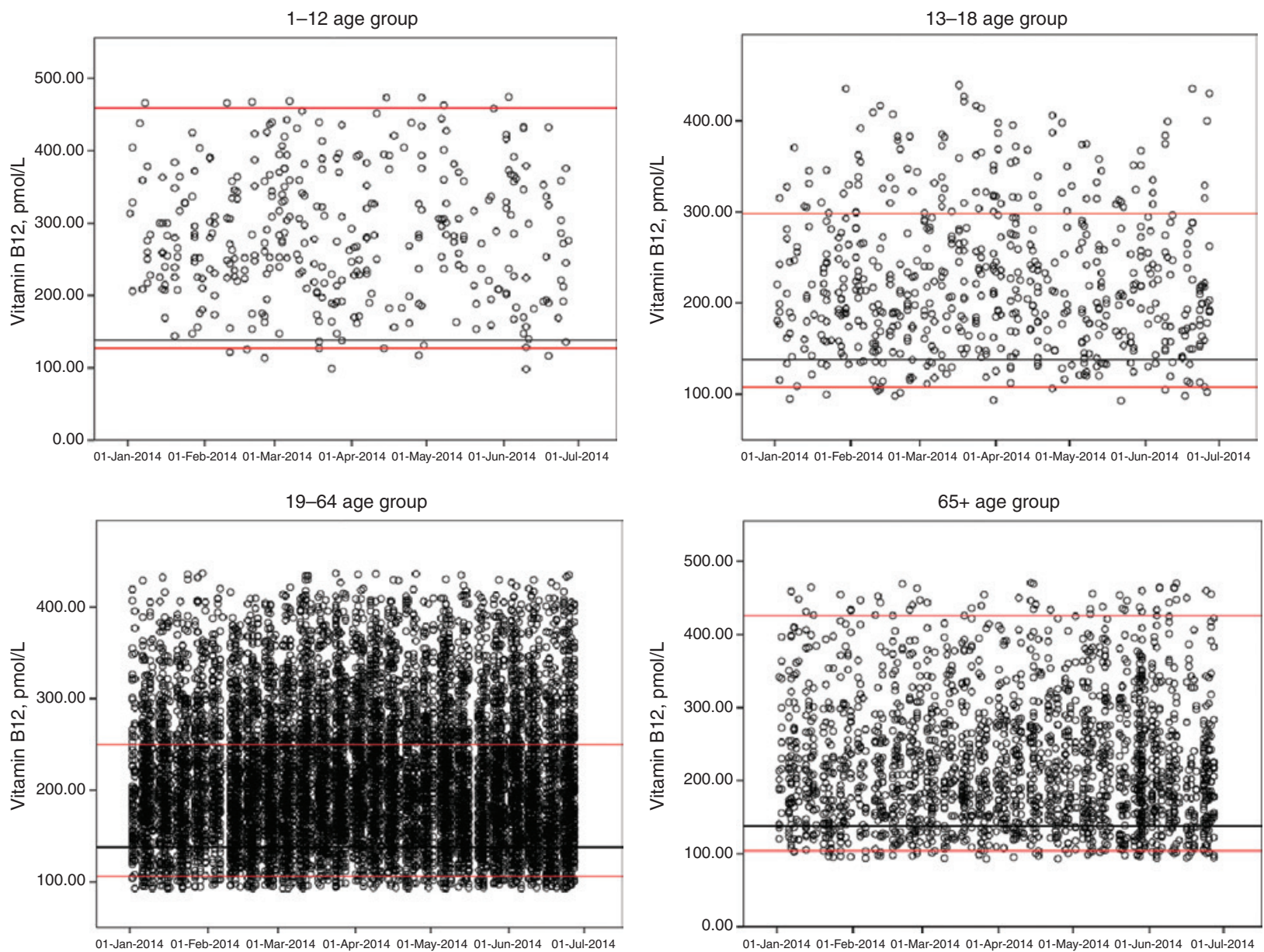

Figure 1: Graphical representation of vitamin B12 test results of all age groups.

At first, we also divided the reference individuals into age and gender groups [19]. The distributions of the group and the subgroups were found to be nonGaussian; therefore, we evaluated the differences of the subgroup RIs (9). We examined the groups' lower limits according to the manufacturer's lower limit and found the smallest difference was in the "1-12" age group. Considering the supplementation of vitamin B12 in this group, we estimated the RIs of the " $1-12$ " and " $13+$ " age groups separately.

Although the reference limits for vitamin B12 are generally presented without partitioning age, our findings show that it can provide more valuable information for decision-making if the RIs are given separately for the "1-12, 13-18, 19-64 and 65+" age groups. We could not find any statistically significant difference between the genders. We estimated the vitamin B12 RIs of our population by using two new approaches, Bhattacharya and Hoffman, and revealed the same intervals as in the indirect approach, which can be seen in Table 2. These methods had similar results with the indirect approach.
There is no accepted cut-off to define vitamin B12 deficiency. The suggestion of the World Health Organization (WHO) for vitamin B12 deficiency is $150 \mathrm{pmol} / \mathrm{L}$.

As a PHL, we provide service to family physicians working at primary healthcare services. Their patients are outpatients, and most of the patients for whom their vitamin B12 is ordered have normal complete blood counts $(14,748$ from 15,455 patients, Table 1$)$. It can be thought that they are screening for vitamin B12 deficiency of individuals admitted to the health centers even if screening average-risk adults for vitamin B12 deficiency is not recommended [20]. However, our findings from our study revealed that the population living in and around Uşak has low vitamin B12 levels with respect to the manufacturer's lower reference limits and the suggested cut-offs for vitamin B12 deficiency. Therefore, the vitamin B12 measurements are appropriate for this geographical area. We could say vitamin B12 deficiency was present $(<106 \mathrm{pmol} / \mathrm{L})$ in the $65+$ age group according to the three estimation methods. As a member of healthcare providers, it is our responsibility to provide information 
Table 3: Vitamin B12 RI studies in our country.

\begin{tabular}{|c|c|c|c|}
\hline \multirow{2}{*}{$\begin{array}{l}\text { Reference; city, } \\
\text { country }\end{array}$} & \multirow[t]{2}{*}{ n (all; M, F) } & \multirow[t]{2}{*}{ Age, year } & \multirow{2}{*}{$\frac{\text { Vitamin B12, pmol/L M, F }(90 \% \mathrm{Cl})}{\text { Assay method }}$} \\
\hline & & & \\
\hline $\begin{array}{l}\text { [13]; İzmir, western } \\
\text { part of Turkey }\end{array}$ & $279 ; 124,155$ & $18-40$ & $\begin{array}{l}74-517^{\mathrm{a}}(74-77,461-613) \\
75-492^{\mathrm{a}}(74-89,451-591) \\
\text { RIA }\end{array}$ \\
\hline $\begin{array}{l}\text { [11]; Bursa, western } \\
\text { part of Turkey }\end{array}$ & $327 ; 143,184$ & $18-40$ & $\begin{array}{l}158-1139^{a}(142-204,1015-1534) \\
235-1473^{a}(136-261,1390-1641) \\
\text { ECLIA }\end{array}$ \\
\hline $\begin{array}{l}\text { [10]; İzmir, western } \\
\text { part of Turkey }\end{array}$ & $274 ; 141,133$ & $20-50$ & $\begin{array}{l}142-953^{a}(129-156,856-1144) \\
\text { ECLIA }\end{array}$ \\
\hline $\begin{array}{l}{[12] ; \text { Erzurum, }} \\
\text { eastern part of } \\
\text { Turkey }\end{array}$ & $402 ; 202,200$ & $18-65$ & $\begin{array}{l}70-368^{\mathrm{a}}(59-79,321-388 \\
71-395^{\mathrm{a}}(56-90,372-413) \\
\text { ECLIA }\end{array}$ \\
\hline $\begin{array}{l}\text { [2]; Konya, central } \\
\text { part of Turkey }\end{array}$ & $2368 ; 512,1856$ & $1-70$ & $\begin{array}{l}75-518^{b} \\
E C L I A\end{array}$ \\
\hline $\begin{array}{l}{[17] ; \text { Düzce, }} \\
\text { northern part of } \\
\text { Turkey }\end{array}$ & $1251 ; 490,761$ & $18-79$ & $\begin{array}{l}117-374(115-119,327-412) \\
117-415(116-118,387-447) \\
\text { ECLIA }\end{array}$ \\
\hline
\end{tabular}

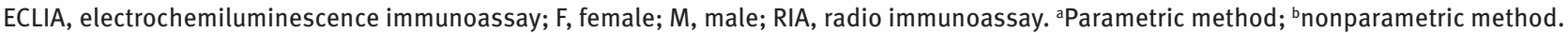

on the health status of our population according to the laboratory test results by the indirect reference estimation method. It is recommended that the initial laboratory assessment of a patient with suspected vitamin B12 deficiency should include a complete blood count and a serum vitamin B12 level [21]. This recommendation is suitable for primary healthcare services in Turkey. However, we suggest that these vitamin B12-deficient individuals should be tested for methyl malonic acid, homocysteine and folic acid [22]. The main challenge for our study was the distance from our laboratory to the FHCs varied from $2 \mathrm{~km}$ to $60 \mathrm{~km}$. The PHL is localized in the center of the city of Uşak that has a population of 180,000 in the western part of Anatolia. There are 21 FHCs in different regions of the Uşak province. The tests are ordered by family physicians, and the specimens are collected by nurses working at the FHCs. All specimens are transported to the PHL 9 times a day under strictly controlled conditions for preanalytical variations. Our findings show that the measurement results of analytes such as vitamin B12 whose blood levels mostly depend on the nutritional status of the individuals should be considered as a national health subject.

\section{Conclusions}

The indirect method is a valuable tool for the estimation and verification of RIs in every clinical laboratory that has a high test capacity. The public healthcare centers have opportunity with their high number of outpatients. The main challenge is that the laboratories have to be familiar with the relevant statistical calculations and big data analysis.

The estimation of the indirect RIs may also provide the status of the population with respect to the analyte. Our results show that the vitamin B12 status of our population needs to be examined by policy makers. The other contribution of such studies is that laboratories can be used as data centers of healthcare institutions.

Policy makers should have a policy to evaluate the laboratory test results in view of the total national health status. Every medical laboratory can add value to healthcare services through studies such as this. In addition to these, laboratories as health data repository centers can provide invaluable and meaningful information for the healthcare policy if the data management system is designed properly and human resources with data mining skills are available.

Author contributions: All the authors have accepted responsibility for the entire content of this submitted manuscript and approved submission.

Research funding: None declared. Employment or leadership: None declared. Honorarium: None declared.

Competing interests: The funding organization(s) played no role in the study design; in the collection, analysis, and interpretation of data; in the writing of the report; or in the decision to submit the report for publication. 


\section{References}

1. Tolan NV, Parnas ML, Baudhuin LM, Cervinski MA, Chan AS, Holmes DT, et al. "Big data” in laboratory medicine. Clin Chem 2015;61:1433-40.

2. Oncel M, Sahin E, Kiyici A, Adam B. Use of laboratory information system data for indirect estimation of reference interval for vitamin B12. LaboratoriumsMedizin 2015;39:437-41.

3. Enli Y. Determination of reference intervals for $18-40$ years old people living in Denizli by using different methods. Turk J Biochem 2004;28:228-45.

4. Jones RD, Haeckel R, Loh TP. Indirect methods for reference interval determination - review and recommendations. Clin Chem Lab Med 2019; 57:20-29.

5. Rannelli L, Watterson R, Pandya R, Leung AA. Vitamin B12 deficiency with combined hematological and neuropsychiatric derangements: a case report. J Med Case Rep 2014;8:1-4.

6. Oh R, Brown DL. Vitamin B12 deficiency. Am Fam Physician 2003;67:979-86.

7. Birch JB, Tukey JW. Exploratory data analysis. J Am Stat Assoc 1978;73:885.

8. Solberg HE. The IFCC recommendation on estimation of reference intervals. The RefVal program. Clin Chem Lab Med 2004:42:710-4.

9. Lahti A. Partitioning biochemical reference data into subgroups: comparison of existing methods. Clin Chem Lab Med 2004;42:725-33.

10. Grossi E, Colombo R, Cavuto S, Franzini C. Indirect methods for reference intervals based on current data. Clin Chem 2006;52:337-8.

11. Akin F, Yavuz H, Bodur S, Kiyici A. Vitamin B12 levels of subjects aged 0-24 year(s) in Konya, Turkey. J Heal Popul Nutr 2014;32:615-22.
12. Papandreou D, Mavromichalis I, Makedou A, Rousso I, Arvanitidou M. Total serum homocysteine, folate and vitamin B12 in a Greek school age population. Clin Nutr 2006;25:797-802.

13. Wahlin Å, Bäckman L, Hultdin J, Adolfsson R, Nilsson L-G. Reference values for serum levels of vitamin B12 and folic acid in a population-based sample of adults between 35 and 80 years of age. Public Health Nutr 2007;5:505-11.

14. Demirin H, Memisogullari R, Ucgun T, Yildirim HA, Celer A, Bulur $S$, et al. Have the reference ranges of anemia parameters like iron, ferritin, vitamin B12 and folate been correctly settled in Turkish adults living in western Black Sea Region? Turk J Biochem 2012;37:356-61.

15. Köseoğlu M, Işleten F, Dursun S, Çuhadar S. Determination of reference intervals of healthy adults aged between $20-50$ years in Izmir. Turk J Biochem 2010;35:215-24.

16. İlçöl YÖ, Aslan D. Determination of reference intervals of the healthy subjects in Bursa. Turk J Biochem 2004;29:183-92.

17. Bakan E, Polat H, Ozarda Y, Ozturk N, Baygutalp NK, Umudum FZ, et al. A reference interval study for common biochemical analytes in eastern turkey: a comparison of a reference population with laboratory data mining. Biochem Medica 2016;26:210-23.

18. Tanyalcin T, Aslan D, Kurtulmus Y, Gökalp N, Kumanlioğlu K. Reference intervals of serum folate and vitamin B12 developed from data of healthy subjects. Accred Qual Assur 2000;5:383-7.

19. CLSI C28-A3. Defining, establishing, and verifying reference intervals in the Clinical Laboratory; Approved Guideline, 3rd ed. CLSI document C28-A3. Wayne, PA: Clinical and Laboratory Standards Institute, 2008.

20. Langan RC, Zawistoski KJ. Update on vitamin B12 deficiency. Am Fam Physician [Internet] 2011;83:1425-30.

21. Langan RC, Goodbred AJ, Luke S, Residency M. Vitamin B12 deficiency: recognition and management. Am Fam Physician 2017;96:384-9.

22. Gilfix M. Brain, vitamin B12 and homocysteine. Can Med Assoc J 2005;173:1360. 